\title{
An Analysis of Skin Pixel Detection using Different Skin Color Extraction Techniques
}

\author{
Gururaj P Surampalli \\ M.Tech (CSE) Student \\ GNDEC Bidar-585401
}

\author{
Dayanand J \\ Asst. Professor \\ GNDEC Bidar-585401
}

\author{
Dhananjay M \\ Asst. Professor \\ GNDEC Bidar-585401
}

\begin{abstract}
Automated skin detection from a captured natural image has wide range of application. Detection of skin area in a given image is done through marking skin and non skin pixels. Process of identification of skin pixel is closely associated with color space being used. To select suitable method to extract skin region has motivated this paper. We are using multiple color spaces in a paper to analyze and compare them. We have the different set of images to compare color space. The results indicate that $\mathrm{YCbCr}$ provide better performance compare to other color space.
\end{abstract}

\section{General Terms:}

Color space, Skin pixel

\section{Keywords:}

Skin pixel detection; Log opponent; HSV; YIQ; YCbCr

\section{INTRODUCTION}

The skin color is useful in most of the recent technologies which include face detection, localization, image content filtering, content aware video compression and image color balancing applications. There are many techniques for skin color modeling and recognition, which are proposed during the past years. Most commonly used is skin segmentation techniques, which involve the classification of individual image pixels into skin and nonskin categories on the basis of pixel color [6]. It is very hard task to extract regions of specific color from a given color image, the color of an object varies with changes in illumination color. It is found in present work that separating illumination from chrominance can produce good result in detecting skin pixel [2]

Generally human skin is characterized by a combination of red and melanin (yellow, brown) and there is somewhat a range of hue for skin and saturation that represent skin pixels [10].

The detection of human skin effected by various external factors, such as types of cameras used to capture the image, light-setting, human race and color spaces [1]. Skin color detection most important process in vision systems, like gesture recognition, region of interest hand tracking, video indexing, face detection, etc [7].

The skin pixel based detection can reduce the search space before high level processing, however this is not an easy task. Skin pixels vary with ambient light, such as color lamps which acts as a filter, brightness and shadows, daylight, etc. Different cameras with variant resolution return different values for the same scene. Skin pixel detection is a cumbersome task. The main aim of skin color detection is to build a decision rule that will differentiate between skin and non-skin region based on pixels values.
Determining skin pixel color involves finding the range of values for which most of the skin pixels would fall in a given color space. The theme of a color space is to facilitate the specification of colors in some standard way, generally accepted manner. A color space includes specification of a coordinate system and the subspace within a system where each color is represented by a single point. In later color spaces the orientation is toward hardware such as color monitors or toward applications where color manipulation is a goal such as the creation of color graphics or animation. Numerous color spaces are used for processing digital images. For some process, one color space may be more appropriate than other color space [7]. Most of the work has been done on finding faces in color images using methods such based on chrominance pesent in image [4], detecting face based on skin color [8] [11] and based on AdaBoost [12].

The block diagram of the skin detection using Log opponent, HSV, YIQ, YCbCr techniques discussed in present paper as shown: Generally, a good skin color model must offer a high

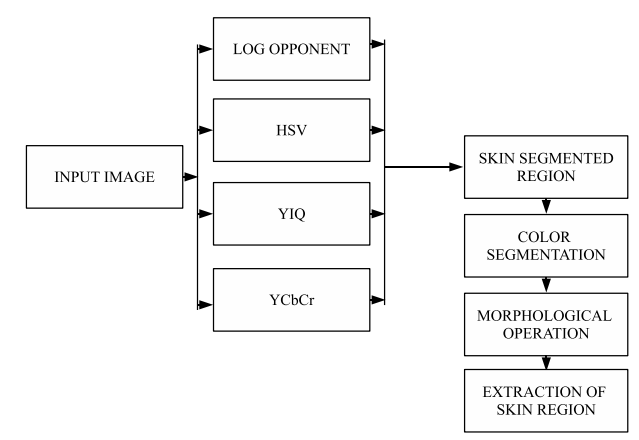

Fig. 1. Block diagram of skin detection

detection rate and a low false positive rate. That is, skin color model must detect more number of skin pixels while minimizing the number of non-skin pixels classified as skin [9].

Pixel classification is complicated and there are number of suggested methods for classifying pixels color as skin or non-skin color in an attempt to achieve the maximum performance [3].

\section{SOME SKIN PIXEL DETECTION TECHNIQUES}

\subsection{Log opponent}

The log opponent color space is similar to the human visual system, the log opponent may be expressed in terms of the two 
opponent hues, yellow-blue and green-red, which will get cancel each other superimposed. Log opponent color space for skin pixel color detection is suggested by Fleck et al [6].

Where $\mathrm{n}$ is a random noise value generated from a uniform distribution over the range $[0,1]$ and the constant 105 is used to scale the range to the interval $[255,255]$.

The algorithm for the log opponent is as follows:

Step 1: Take input image, which contains RGB values.

Step 2: Intensity in the image is calculated by multiplying the parameter value $(0.596,-0.274,-0.322)$ to the RGB components. Step 3: The hue present in the image is calculated by taking inverse tangent of the $\left(R_{g}, B_{y}\right)$.

Step 4: Check whether intensity lies between $(20,90)$ and hue lies between $(100,150)$ [6].

Step 5: If the pixel satisfies Step: 4, the detected pixel is skin pixel. Otherwise the pixel detected is not skin pixel.

The result using log opponent as shown

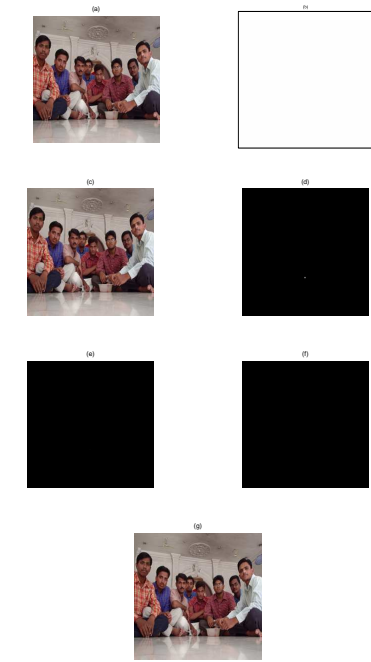

Fig. 2. (a) Input image. (b) Segmented. (c) Color segmented image. (d) Edge detection. (e) Binarilization. (f) Morphological operation.

(g) Skin detected.

From the results, it can be observed that segmented image is white because the all pixel present in image considered as skin pixel. Hence, futher processing steps also fail to give correct result.

\subsection{HSV (Hue, Saturation, Value)}

The HSV color space defines color with intuitive values, based on the artist idea of characteristics such as tint, saturation and tone. It was introduced when it is needed to mention color properties numerically. Hue defines the dominant color as defined by wavelength, let us for instance the difference between red and yellow colors. Saturation is the measures of colorfulness area in proportion to the brightness on the area, such as the distinction between red and pink color. The value indicates the color luminance, the distinction between a dark red and a light red. In skin detection, the numeric value of component is discarded to eliminate the undesirable effect of not evenly distributed illumination [6].

There are some of the studies, which shows that HSV is invariant to highlights at white light sources, to matte surfaces, and ambient lighting. However, hue non continuities and the computation of the luminance component conflict badly with the properties of color vision. The cyclic behavior of Hue-Saturation spaces also makes it inconvenient for parametric skin color models that need a rigid cluster of skin performance, for optimum performance. The selected range of $\mathrm{H}$ control segmentation ton reddish colors and the saturation range selected ensures the exclusion of pure red and very dark red colors, both of which are caused by small variations in lighting conditions. The threshold on value $(\mathrm{V})$ is introduced to reject dark colors.

The algorithm for the HSV is as follows:

Step 1: Take input image, which contains RGB values.

Step 2: Convert RGB to HSV components.

Step 3: Check whether saturation lies between $(0.20,0.75)$, value greater than 0.35 and hue lies between $(0,25)$ [6].

Step 4: If the pixel satisfies Step: 3, the detected pixel is skin pixel. Otherwise the pixel detected is not skin pixel.

The result using HSV as shown
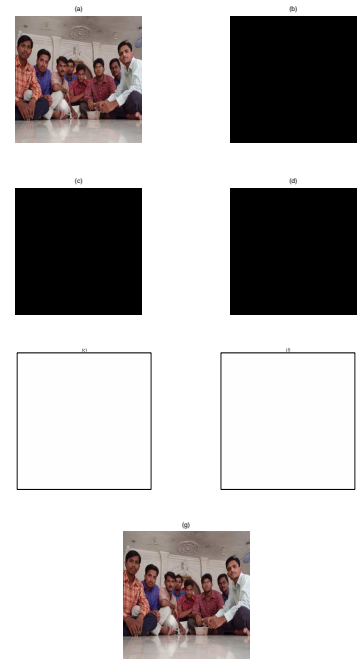

Fig. 3. (a) Input image. (b) Segmented. (c) Color segmented image. (d) Edge detection. (e) Binarilization. (f) Morphological operation. (g) Skin detected.

The results shown indicate that all the pixel in an image considered as non skin pixel, therefore a black image. The black image converted into binary image (f), which is white. Hence, morphological image is also white.

\subsection{YIQ (Luma, Inphase, Quadrature)}

It is also known as modified log opponent technique [6]. The YIQ system is intended to take advantage of human colorresponse characteristics. The algorithm for the YIQ is as follows:

Step 1: Take input image, which contains RGB values.

Step 2: Intensity in the image is calculated by multiplying the parameter value $(0.5957,0.2745,-0.3213)$ to the RGB components [6].

Step 3: Check whether intensity lies between $(20,90)$ and hue lies between $(100,150)$ [6].

Step 4: If the pixel satisfies Step: 3, the detected pixel is skin pixel. Otherwise the pixel detected is not skin pixel.

The result using YIQ as shown

The results are similar to log opponent, it can be observed that segmented image is white because the all pixel present in image considered as skin pixel. Hence, futher processing steps also fail to give correct result. 


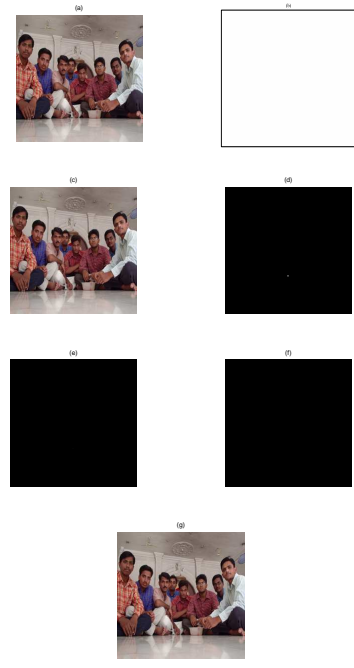

Fig. 4. (a) Input image. (b) Segmented. (c) Color segmented image. (d) Edge detection. (e) Binarilization. (f) Morphological operation. (g) Skin detected.

\subsection{YCbCr (Luma, Blue Chroma, Red Chroma)}

$\mathrm{YCbCr}$ color space has been defined to meet the increasing requirements of digital algorithms in handling video information and has become the most frequently used color space in digital videos. YCbCr is consist of three components, two of them is of chrominance and one is of luminance[5].

In order to optimize the performance of skin color clustering, the present work uses $\mathrm{YCbCr}$ space to build a skin color model, since it is also known that, as the chrominance components are almost independent of luminance component in the space there are non-linear relations between chrominance $(\mathrm{Cb}, \mathrm{Cr})$ and luminance $(\mathrm{Y})$ of skin pixel color in the high and low luminance region. As in RGB space, the triple component $(\mathrm{r}, \mathrm{g}, \mathrm{b})$ expresses not only color but also luminance. Luminance may change across a person's face due to the ambient lighting and is not a reliable measure in discriminating skin from non-skin region.

$\mathrm{YCrCb}$ is actually an encoded nonlinear RGB signal, commonly used by European television studios and for image compression work. Color is represented by luma (that is luminance, computed from nonlinear RGB), it is constructed as a weighted sum of the RGB values, and two color difference values $\mathrm{Cr}$ and $\mathrm{Cb}$ that are calculated by subtracting luma from RGB red and blue components. The conversion simplicity and explicit separation of luminance and chrominance components makes this color space attractive for skin color modeling. In $\mathrm{YCbCr}$ color space, the two chroma components $\mathrm{Cr}$, and $\mathrm{Cb}$ can be efficiently used to define explicitly skin region. The thresholds be selected as (Crmax; Crmin) and (Cbmax; Cbmin) , a pixel value is classified as skin pixel, if the values $(\mathrm{Cr}, \mathrm{Cb})$ fall within the thresholds. The luminance has to be removed from the color representation in the chromatic color space. Chromatic colors known as "pure" colors in the absence of luminance.

The $\mathrm{YCbCr}$ conversion from RGB color space can be accomplished by following matrix.

$$
\left[\begin{array}{c}
Y \\
C b \\
C r
\end{array}\right]=\left[\begin{array}{ccc}
1 & 1 & 1 \\
0.148 & -0.291 & 0.439 \\
0.439 & -0.368 & -0.071
\end{array}\right]\left[\begin{array}{l}
R \\
G \\
B
\end{array}\right]+\left[\begin{array}{c}
16 \\
128 \\
128
\end{array}\right]
$$

For the skin pixel using $\mathrm{YCbCr}$, red chrominance value lies between $(140,165)$, blue chrominance value lies between $(140$, $195)$ and hue value lies between $(0.01,0.1)$. The result using $\mathrm{YCbCr}$ as shown

From results of $\mathrm{YCbCr}$, it is found that the segmented and color

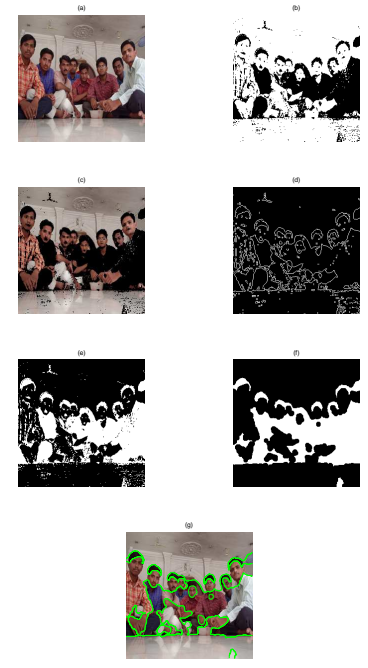

Fig. 5. (a) Input image. (b) Segmented. (c) Color segmented image. (d) Edge detection. (e) Binarilization. (f) Morphological operation. (g) Skin detected.

segmented images are formed on the basis of skin pixel. The further processing is also carried out, the skin region detected is shown in image $(\mathrm{g})$.

\section{RESULTS AND DISCUSSION}

\subsection{Single image analysis}

Consider the image for applying all the techniques discussed it is found that $\mathrm{YCbCr}$ is more capable in finding more valid skin pixel as shown.

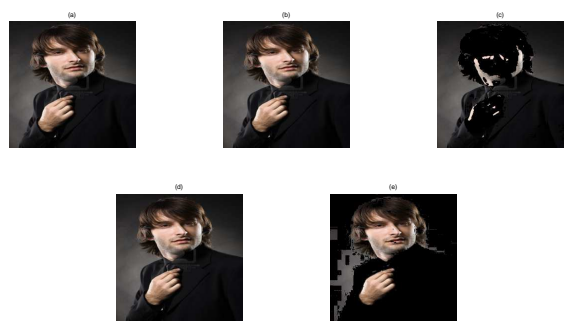

Fig. 6. Input image and results of Log opponent HSV YIQ YCbCr

For single image it has been observed from the results that, the $\mathrm{YCbCr}$ technique is able to recognize skin pixel more as compared to other techniques. The HSV technique also produces some good result. As shown below.

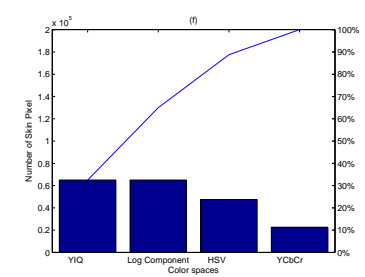

Fig. 7. Graph shows Number of skin pixel detected in different colorspaces

The graph displays a pareto chart, where the pixel values in 
the vector y are drawn as bars in descending order. Each bar is labeled with the associated skin detection technique. The line above the bars shows the cumulative percentage. It is observed that with several test images, the region of skin can be detected efficiently by using YCbCr technique. Log opponent, HSV and YIQ fails to detect the correct skin pixel. The table 1 below shows number of skin pixel detected in images shown in results.

Table 1. Number of Skin Pixel Detected for Test Images

\begin{tabular}{|c|c|c|c|c|}
\hline Image & Log opponent & HSV & YIQ & YCbCr \\
\hline 1 & 65025 & 0 & 65025 & 9352 \\
\hline 2 & 65025 & 16092 & 65025 & 27552 \\
\hline 3 & 65025 & 6 & 65025 & 21474 \\
\hline 4 & 65025 & 0 & 65025 & 1546 \\
\hline 5 & 65025 & 0 & 65025 & 5916 \\
\hline 6 & 65025 & 39378 & 65025 & 9583 \\
\hline 7 & 65025 & 119 & 65025 & 22628 \\
\hline 8 & 65025 & 251 & 65025 & 19980 \\
\hline 9 & 65025 & 21 & 65025 & 8708 \\
\hline 10 & 65025 & 0 & 65025 & 17638 \\
\hline
\end{tabular}

\subsection{Comparison of Log Opponent, HSV, YIQ and YCbCr Techniques}

When each technique is applied to set of test images, following graph can drawn indication number of skin pixel and non skin pixel present in the image.
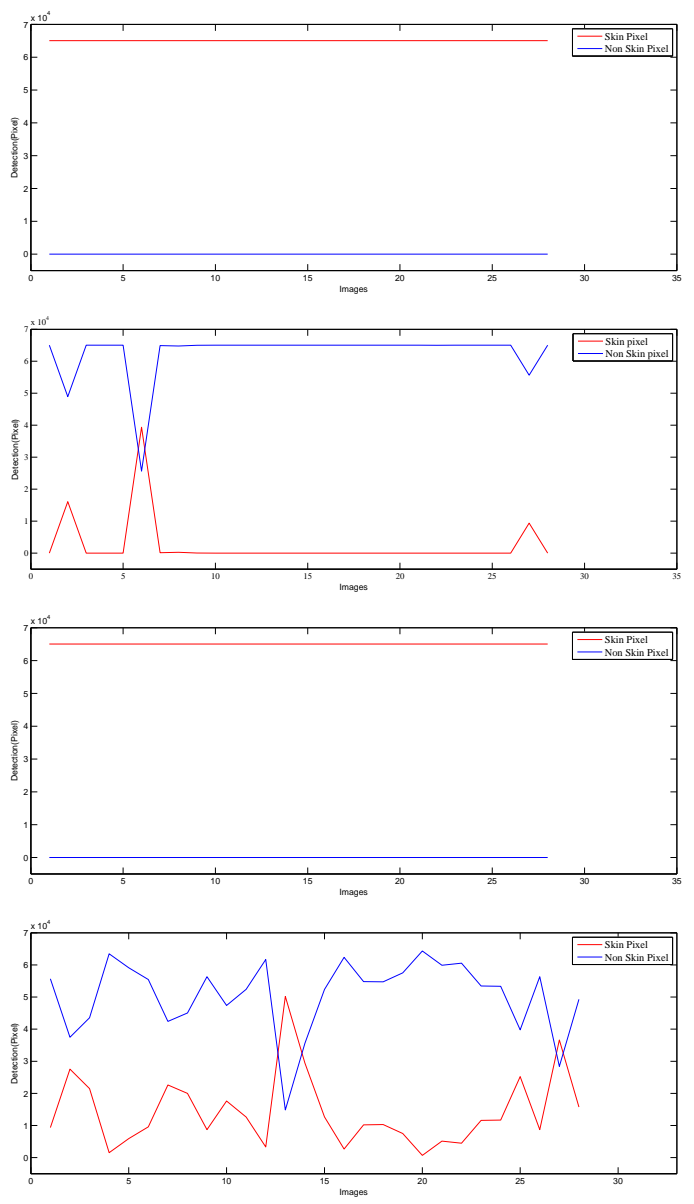

Fig. 8. Comparison of Log opponent, HSV, YIQ, YCbCr
From above graphs the following can be determined:

\section{-Log opponent:}

It has been observed that log opponent technique provides the result in which it considers the all pixel present in the image as skin pixel. The graph shows the blue bars indicating skin pixel present in the some test images processed. Log opponent technique fails to provide good result as observed but the improvement can be achieved by changing the parametric values.

\section{-HSV:}

The HSV technique provide good result as compared to the $\log$ opponent, the red bars represents the non skin pixels present in the test image. It is observed that the region of skin area provided by this technique is not satisfactory.

\section{-YIQ:}

YIQ results are as shown. By observation the results are similar to the $\log$ opponent. That is the number of pixel present in the image considering as skin pixel shown by blue bars. Blue bars indicate number of skin pixel present in an image.

\section{$-\mathrm{YCbCr}$}

The study and experimental results proves that $\mathrm{YCbCr}$ technique or color space provides correct skin pixel among other discussed. The result shows number of pixel detected as skin and non skin pixel.

As per the above set of graphs and by the observation, it is found that Log opponent, YIQ are fail to detect the exact skin pixel present in the test images. Hence values which are provided by these techniques are not adequate. For the image of size $255 \mathrm{x}$ 255 providing result 65025 , that is whole area of the image is considering as skin area which not true.

In another case where $\mathrm{HSV}$ and $\mathrm{YCbCr}$ are providing good result. Hence comparing the two techniques on the basis of there detection ability, following graph can be drawn.
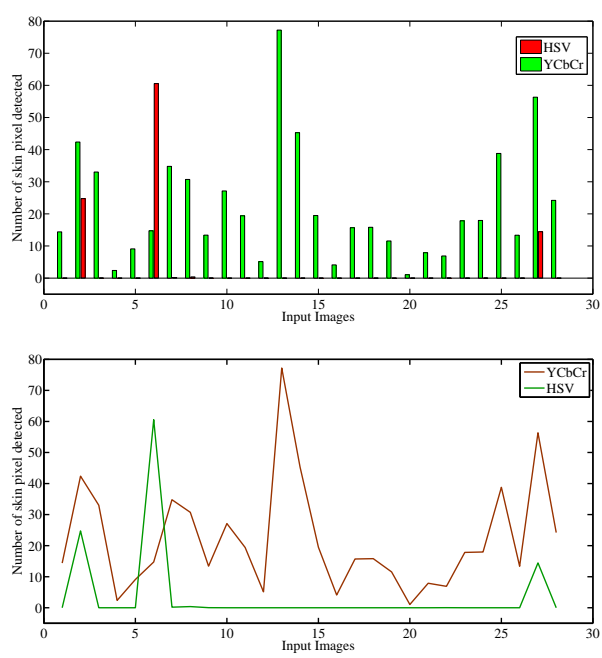

Fig. 9. Comparison of HSV, YCbCr techniques

From figure 9, it is observed that image 6 which is giving high value for the HSV as compared to YCbCr, this effect may be because of wrong image condition as shown below. Except in all cases the $\mathrm{YCbCr}$ is giving correct result. The skin pixel detection efficiency of $\mathrm{HSV}$ is $3.57 \%$ and for $\mathrm{YCbCr}$ is $96.42 \%$ for the set of test images considered from data base. That is detection 
Table 2. Percentage of skin region detected for some images with size 255 x 255

\begin{tabular}{|c|c|c|c|c|}
\hline Image & HSV & HSV in $\%$ & YCbCr & YCbCr in $\%$ \\
\hline 1 & 0 & 0 & 9352 & 14.384 \\
\hline 2 & 16092 & 24.747 & 27552 & 42.374 \\
\hline 3 & 6 & 0.009 & 21474 & 33.024 \\
\hline 4 & 0 & 0 & 1546 & 2.377 \\
\hline 5 & 0 & 0 & 5916 & 9.098 \\
\hline 6 & 39378 & 60.558 & 9583 & 14.737 \\
\hline 7 & 119 & 0.183 & 22628 & 34.798 \\
\hline 8 & 251 & 0.386 & 19980 & 30.726 \\
\hline 9 & 21 & 0.032 & 8708 & 13.391 \\
\hline 10 & 0 & 0 & 17638 & 27.124 \\
\hline
\end{tabular}

efficiency of $\mathrm{YCbCr}$ is more compared to HSV, Log opponent and YIQ. The percentage of skin detected for some images of
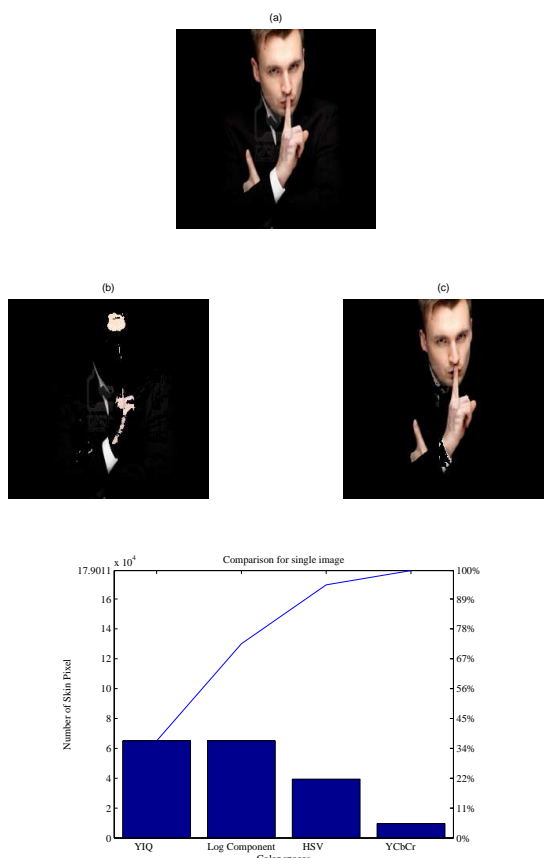

Fig. 10. (a). Input image, Output of (b). $\mathrm{HSV}$, (c). YCbCr, (d). Comparison graph showing number of skin pixel detected

size $255 \times 255$ from database by using $\mathrm{HSV}$ and $\mathrm{YCbCr}$ shown in table 2. The reason for percentage change in the HSV for image 6 as shown in figure 10 .

\section{CONCLUSION}

Skin detection plays very important and crucial part in many biometric systems. The work has been carried out to understand and analyze the log opponent, HSV, YIQ and YCbCr skin pixel detection techniques. Each technique perform the pixel detection using different set of parameters for detecting skin pixel and non skin pixel.

The proper analysis is carried out during the present work, which involve applying each technique to set of images present in database. The comparison of each technique for the single image is performed. Further work extends for comparing the log opponent, HSV, YIQ, and $\mathrm{YCbCr}$ for skin pixel detection ability. Results show that log opponent, YIQ fails to detect the correct skin pixel. Hence analysis is emphasized on HSV, YCbCr. During the comparison of $\mathrm{HSV}, \mathrm{YCbCr}$ it is found that skin pixel detection efficiency of $\mathrm{HSV}$ is $3.57 \%$ and for $\mathrm{YCbCr}$ is $96.42 \%$ for the set of images used from database.

\section{ACKNOWLEDGMENTS}

I owe my thanks much more than the words can express to my parents and friends without whose co-operation and inspiration this paper would have been a distant dream.

\section{REFERENCES}

[1] E Angelopoulou. Understanding the color of human skin. In Proc. SPIE Conf. On Human Vision and Electronic Imaging VI (SPIE), 4299:243-251, 2001.

[2] J Brand, S Mason, M Roach, and M Pawlewski. Enhancing face detection in colour images using a skin probability map. Int. Conf. on Intelligent Multimedia, Video and Speech Processing, pages 344-347, 2001.

[3] Cynthia A Brewer. Color use guidelines for data representation. Alexandria: American Statistical Association, pages 55-56, 1999.

[4] D N Chandrappa, M Ravishankar, and D R RameshBabu. Automated detection and recognition of face in a crowded scene. International Journal of Computer and Network Security, 2(6):65-70, June 2010.

[5] D N Chandrappa, M Ravishankar, and D R RameshBabu. Face detection in color images using skin color model algorithm based on skin color information. IEEE, pages 254258,2010 .

[6] Tarek Abd El-Hafeez. A new system for extracting and detecting skin color regions from pdf documents. International Journal on Computer Science and Engineering(IJCSE), 2(9):2838-2846, 2010.

[7] B Hajar, E Sanaa, J Abdelilah, and A Driss. Recognition of adult video by combining skin detection features with motion information. IEEE, 2010.

[8] Jiang Qiang-rong and Li Hua-lan. Robust human face detection in complicated color images. IEEE Trans, 2010.

[9] S Sanjay, D S Chauhan, V Mayank, and S Richa. A robust skin color based face detection algorithm. Tamkang Journal of Science and Engineering, 6(4):227-234, 2003.

[10] M R Tabassum, A U Gias, M M Kamal, H M Muctadir, M Ibrahim, A K Shakir, A Imran, S Islam, M G Rabbani, S M Khaled, M S Islam, and Z Begum. Comparative study of statistical skin detection algorithms for sub-continental human images. Institute of Information Technology, University of Dhaka, pages 1-8.

[11] Randazzo Vincenzo and Usai Lisa. An improvement of adaboost for face-detection with motion and color information. IEEE 14th International Conference on Image Analysis and Processing (ICIAP), 2007.

[12] Yan-Wen Wu and Xue-Yi Ai. Face detection in color images using adaboost algorithm based on skin color information. IEEE computer society, 2008. 